\title{
Delayed-Choice Experiments and the Metaphysics of Entanglement
}

the date of receipt and acceptance should be inserted later

\begin{abstract}
Delayed-choice experiments in quantum mechanics are often taken to undermine a realistic interpretation of the quantum state. More specifically, Healey has recently argued that the phenomenon of delayed-choice entanglement swapping is incompatible with the view that entanglement is a physical relation between quantum systems. This paper argues against these claims. It first reviews two paradigmatic delayed-choice experiments and analyzes their metaphysical implications. It then applies the results of this analysis to the case of entanglement swapping, showing that such experiments pose no threat to realism about entanglement.
\end{abstract}

Keywords Delayed-choice experiments · Double-slit experiment · Entanglement swapping · Quantum eraser · Realism

\section{Introduction}

Entangled states of quantum systems have played an important role in debates on the metaphysics of contemporary science. Teller [25], French [8], Maudlin [15] and Esfeld [6] all base metaphysical claims on the quantum mechanical description of entangled two-particle states. Glossing over the substantial differences between these accounts, their general thrust is that quantum mechanics pushes us away from a metaphysics of individual things towards a metaphysics of relations, relations which do not supervene on the intrinsic properties of their relata. Of course, such arguments presuppose that what appears as the description of an entangled state in the quantum formalism actually represents a real relation in the world. This presupposition derives support from the fact that measurements on entangled states exhibit

M. Egg

Department of Philosophy, University of Lausanne, CH-1015 Lausanne, Switzerland E-mail: matthias.egg@unil.ch 
significant correlations, as demonstrated, for example, in experimental tests of Bell's inequality. Furthermore, the flourishing of quantum information theory in the last two decades has resulted in a rather dramatic shift of attitude towards entanglement: While it was once considered a bizarre consequence of the quantum formalism with little importance outside of philosophical debates, entanglement has now come to be recognized as a physical resource which can be experimentally manipulated in various ways ${ }^{1}$

In the present paper, I will investigate whether this manipulability really supports a realist stance on entanglement relations. More specifically, I will focus on one particular kind of such manipulations, namely entanglement swapping. In this process, two pairs $(A, B)$ and $(C, D)$ of entangled particles are created by two independent sources. If one then performs the right kind of joint measurement on particles $B$ and $C$, the pair $(A, D)$ enters into an entangled state even though $A$ and $D$ have never interacted with each other. Interestingly, this phenomenon has given rise to contradicting ontological conclusions. On the one hand, Rob Clifton [3, p. S163] takes it to support a realistic view of entanglement: "It appears that there is sufficient substantiality to entanglement that it can be swapped from one pair of particles to another". On the other hand, Richard Healey [9 studies an entanglement-swapping experiment in which the measurement on $B$ and $C$ is performed after $A$ and $D$ have been detected. Since this seems to imply that entanglement can be transferred to a pair of particles which no longer exists, Healey concludes:

The delayed-choice entanglement-swapping experiment reinforces the lesson that quantum states are neither descriptions nor representations of physical reality. In particular, it undermines the idea that ascribing an entangled state to quantum systems is a way of representing some new, non-classical, physical relation between them ${ }^{2}$, p. 759]

Obviously, the "delayed-choice" clause plays a central role here. I will therefore begin my investigation with a brief reminder of the simple and well-known delayed-choice double-slit experiment, assessing its impact on realism about the state of the quantum system (section 2). A more sophisticated (and more radical) version of delayed choice, the so-called quantum eraser, will be discussed in section 3. The case of the quantum eraser is important because it introduces the idea of sorting experimental results into different subensembles, thus raising the question whether these subensembles correspond to real properties of the system. In section 4 I will apply these considerations to the entanglement-swapping experiment and show that if the experiment is carried out in a delayed-choice setting, no actual entanglement swapping occurs.

\footnotetext{
1 For an extensive review of this development and the current state of play, see $[12]$.

2 Healey advances this argument in the context of his pragmatist approach to quantum theory, which I will not discuss here. Neither will I discuss the positions of those who take quantum information theory to support an epistemic or informational (as opposed to metaphysical) view of the quantum state. See [26] for a critique of these approaches.
} 
This will, in section 5, lead to the conclusion that delayed-choice entanglement swapping does not undermine realism about entanglement relations.

\section{Delayed Choice in the Double-Slit Experiment}

The double-slit experiment is probably the best known illustration of the basic mystery of quantum mechanics. If quantum particles (e.g., electrons in a sufficiently coherent state) are sent through a double slit, a characteristic interference pattern appears on the screen behind the two slits. However, this pattern disappears as soon as one tries to detect through which of the two slits each electron passed. It thus seems that the electrons either behave as waves (where "wave behavior" means passing through both slits and producing an interference pattern) or as particles (where "particle behavior" means passing only through one slit and displaying no interference), depending on the kind of experiment we choose to perform. (In the following, I will refer to the two kinds of experimental arrangements as "DS" (for "double slit") and "WW" (for "which way"), respectively.) This is already puzzling enough, but further puzzlement is added by the insight that the decision to perform either a DS or a WW experiment can be taken after the electron has passed through the double slit. It was John A. Wheeler who introduced this idea of a delayed choice, and he took it to imply that "the past has no existence except as it is recorded in the present", and that "[t]he universe does not 'exist, out there,' independent of all acts of observation" [28, p. 41].

It is not hard to see how delayed-choice experiments can lead to such antirealistic conclusions. If we think of the electron as traveling from the source to the double slit and then to the screen where it is detected, a natural question to ask is whether the electron behaved as a wave or as a particle at the time it traveled through the double slit. (That electrons are disposed to behave in either of the two ways ${ }^{3}$ is already known from DS and WW experiments without delayed choice.) Now if the type of experiment (DS or WW) is fixed in advance, this determines the behavior of the electron, and a unique story about its wave- or particle-like nature can be told for each type of experimental setup. However, in the delayed-choice case, the experimenttype is not yet fixed at the time the electron is at the double slit, so it seems that there is simply no fact of the matter as to whether the electron passes through both slits (as waves do) or through only one slit (as particles do).

It is thus clearly impossible to tell a simple realistic story about what happens at the double slit in a delayed-choice experiment, if by "realistic" we mean that the story should attribute a definite, observer-independent behavior to the electron. More sophisticated realistic stories remain, of course, possible, but they do not come without a cost. In the next section, I will argue

\footnotetext{
3 An anonymous referee has reminded me of the fact that there is also a continuous spectrum of intermediate cases, parametrized by the degree to which WW information is obtained (see [7, chap. 1] for a classic discussion). This might be seen as an additional problem for a naive "either-wave-or-particle" view, but I am here only interested in the specific problems arising from the delayed-choice setting. For this purpose, it suffices to look at the two extreme cases of a pure DS and a pure WW experiment.
} 
for such a story, based on the formalism of standard quantum mechanics. I will therefore not have much to say about non-standard quantum theories, such as Bohmian mechanics or the Ghirardi-Rimini-Weber (GRW) theory. But I conclude the present section with some brief remarks about these two theories, in order to illustrate to what extent the delayed-choice double slit complicates the realist's ontological commitments.

At first sight, it seems that Bohmian mechanics has a straightforward answer to the question of what happens at the double slit: Being a particle theory, Bohmian mechanics clearly tells us that each electron goes only through one slit. But it also tells us that the movement of the particle is determined by the wave function, and this raises the tricky question of the ontological status of the latter. Some versions of the theory interpret the wave function as a physical entity which literally guides the particles, or they introduce a so-called quantum potential which gives rise to non-classical forces acting on them. But due to some problems of these interpretations, there is now a tendency among Bohmians to regard the wave function no longer as a physical entity, but merely as a component of the law according to which the particles move [4. However, in their detailed analysis of delayed-choice experiments from a Bohmian perspective, Hiley and Callaghan [10 manage to avoid a commitment to very bizarre particle trajectories only by relying explicitly on the physical reality of the wave function and the quantum potential, so there is reason to doubt that these entities can really be cut off from the ontology of Bohmian mechanics without a loss.

An alternative way to tell a realistic story about the double slit is given by the GRW theory, which adds spontaneous collapses of the wave function to the Schrödinger evolution. But, as argued by Allori et al. 11 (see also [16, pp. 229-238]), this theory is also ambiguous in its ontological commitments. In one version, the wave function describes a matter density in space. Again, this seems to suggest a straightforward solution to our problem: The matter density, being a spatially extended field, (almost) always passes through both slits and collapses to a particle-like object only upon interaction with the detecting screen. The fact that the experimental setup can be chosen after the matter wave has passed the double slit then poses no particular problem. However, according to this story, the result of a WW experiment must be regarded as outright illusory: Even though it looks as if the electron went only through one slit (the experiment telling us which one), the fact is that it went through both. An even more severe illusion takes place according to the second version of the GRW theory, which is merely committed to the existence of some events in space-time (the flashes), corresponding to the spontaneous collapses of the wave function. In this picture, contrary to what we might take as an unquestionable truth about any double-slit experiment, nothing at all travels from the source to the screen.

\section{The Quantum Eraser}

In the experiments discussed so far, the DS/WW decision is taken after the electron has passed through the double slit, but it obviously has to be taken 


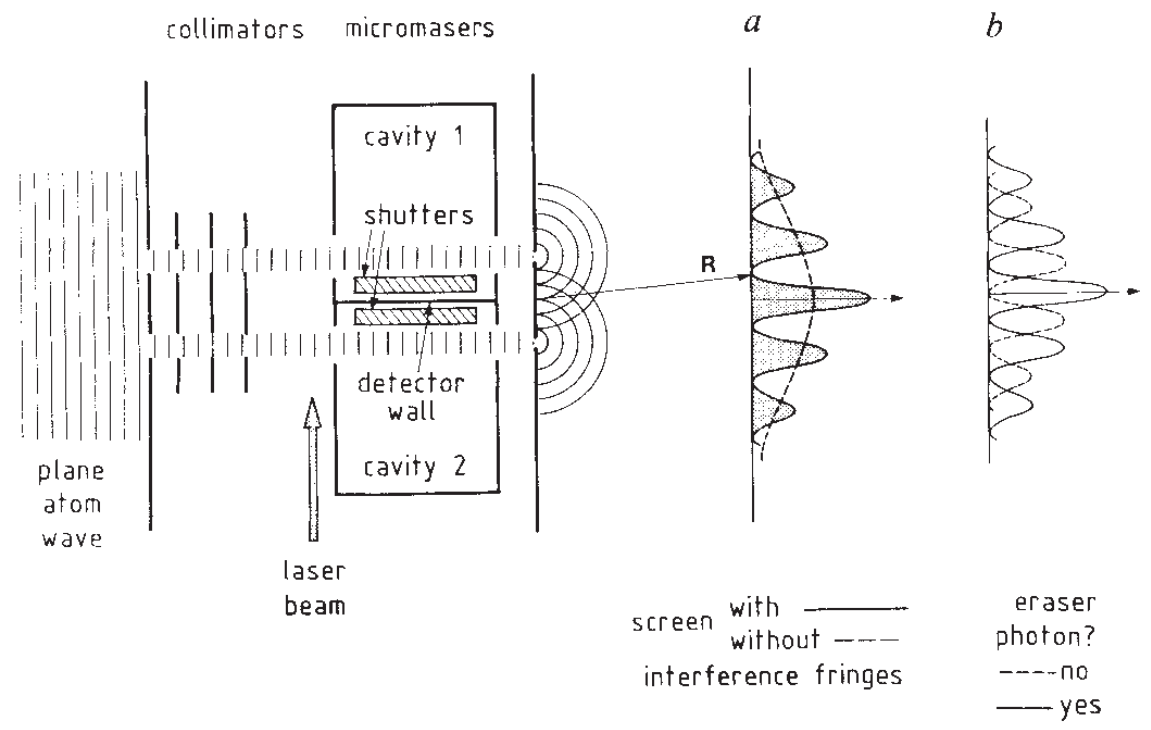

Fig. 1 The quantum eraser thought experiment [23, p. 115].

before the electron is detected. Using a quantum eraser [22, even this restriction can be removed. Consider the thought experiment by Scully et al. [23] depicted in figure 1. In a double-slit experiment with atoms, we place a micromaser cavity in front of each slit. The cavities are designed such that excited atoms passing through them inevitably decay into the ground state by emitting a photon. So for each atom, the corresponding photon emitted in one of the cavities provides us with WW information. However, this information (or more precisely: the possibility of obtaining this information) can be "erased" by opening the shutters which separate the two cavities from a thin-film photodetector placed between them. Since this "detector wall" does not discriminate between photons coming from one or the other cavity, one can then no longer obtain WW information, and a situation resembling the original DS configuration is reestablished. So the experimenter has two options: He can either leave the shutters in place and detect which of the cavities contains the photon, thereby obtaining WW information, or he can open the shutters, allowing the photon to interact with the detector wall without yielding WW information. Note that he can (in principle) decide between these two options after the atom is detected at the screen.

But one might ask how this can really be a choice between a DS and a WW scenario, given that the two scenarios should lead to radically different distributions of atoms on the screen (displaying interference fringes in one case but not in the other). Surely the pattern on the screen can not be changed retroactively? Well, in a certain sense, it can. To see how, a closer look at the quantum mechanical description of the atom-photon-system is necessary ${ }^{4}$ If we denote the photon state by $|1\rangle$ or $|2\rangle$, depending on whether

4 For the following, I adopt the notation of [5]. 
the photon is in cavity 1 or 2 , and the spatial wave function of an atom coming through one of the two slits by $\psi_{1}(x)$ and $\psi_{2}(x)$, respectively, the state of the system after the atom has passed the slits is

$$
|\Psi\rangle(x)=\frac{1}{\sqrt{2}}\left[|1\rangle \psi_{1}(x)+|2\rangle \psi_{2}(x)\right] .
$$

The probability density $\|\Psi(x)\|^{2}$ associated with this state has vanishing interference terms, because $|1\rangle$ and $|2\rangle$ are orthogonal to each other. Therefore, the distribution of atoms on the screen shows no interference fringes, which is what we expect for a WW experiment. Now let us see what happens if the shutters are opened to erase the WW information. As mentioned above, the detector wall does not discriminate between the $|1\rangle$ and the $|2\rangle$ state. However, it does (maximally) discriminate between the symmetric and the antisymmetric superposition states

$$
|+\rangle=\frac{1}{\sqrt{2}}[|1\rangle+|2\rangle] \text { and } \quad|-\rangle=\frac{1}{\sqrt{2}}[|1\rangle-|2\rangle] .
$$

As a consequence, the detector records only photons in the $|+\rangle$ state and ignores photons in the $|-\rangle$ state. Introducing the corresponding symmetric and antisymmetric states of the atom

$$
\psi_{ \pm}(x)=\frac{1}{\sqrt{2}}\left[\psi_{1}(x) \pm \psi_{2}(x)\right]
$$

we can rewrite $(1)$ as

$$
|\Psi\rangle(x)=\frac{1}{\sqrt{2}}\left[|+\rangle \psi_{+}(x)+|-\rangle \psi_{-}(x)\right] .
$$

Since this is just another way of expressing the same state $|\Psi\rangle(x)$, the probability distribution $\|\Psi(x)\|^{2}$ is still the one corresponding to the WW setup. But if we restrict our attention to those atoms for which the photodetector records a photon, the contribution from $|-\rangle \psi_{-}(x)$ vanishes and the probability distribution becomes

$$
P_{+}(x)=\left|\psi_{+}(x)\right|^{2}=\frac{1}{2}\left|\psi_{1}(x)+\psi_{2}(x)\right|^{2},
$$

which simply corresponds to the result of a DS experiment, displaying the usual interference fringes. Conversely, selecting atoms for which the detector does not record a photon yields $P_{-}(x)=\left|\psi_{-}(x)\right|^{2}$, which corresponds to the complementary "anti-fringe" interference pattern (see figure 1b). So each atom can be assigned to one of the four subensembles "1", "2", "+" and "-" in the following way: By measuring the photons in the WW configuration (shutters closed) we classify the atoms into the subensembles 1 and 2, by measuring the photons in the DS configuration (shutters open), we carry out $\mathrm{a}+/-$ classification.

To assess the metaphysical significance of delayed choice in this context, we now need to ask to what physical property (if any) the sorting into subensembles corresponds. Focusing for the moment on subensembles 1 and 
2, the answer seems obvious: All atoms in subensemble 1 went through the first slit, the atoms in subensemble 2 through the second one. Englert et al. 5 , p. 328] endorse this view, but they add an antirealistic twist: "The '... went through ...' is not a statement about the atom's past". This reinterpretation of everyday language is motivated by their "minimalistic interpretation" of the quantum state, which I will not further discuss (see footnote 2 above). In a closely related proposal, Mohrhoff [19] invokes a kind of retrocausation, according to which the present determines the past of the atoms. (Notice the similarity to Wheeler's above-mentioned view that the past's existence depends on its being recorded in the present.) This commitment to retrocausation renders Mohrhoff's "reality-of-phenomena point of view" rather unattractive, but one might be willing to accept this consequence. What one should not accept is Mohrhoff's claim that "retrocausation is a necessary feature of any realistic interpretation of the quantum formalism" [19. p. 332]. He reaches this conclusion by (allegedly) showing that the alternative "reality-of-states point of view" is not viable 5 Here is his argument:

Adherents to the reality-of-states view thus find themselves faced with a dilemma. If they ... deny the possibility of retrocausation, they must insist that it is only as if the atom had traveled through the first cavity or only as if it had been retroactively furnished with a definite phase relation. They cannot say that the atom really was in the state $\psi_{1}$ (or $\psi_{2}$, or $\psi_{+}$, or $\psi_{-}$, as the case may be). And so they find themselves compelled to foreswear realism and embrace operationalism. And if they stick to realism, they will have to drop the as if's and accept the reality of retrocausation. (ibid.)

But the first thing a reality-of-states view should take seriously is the fact that $|\Psi\rangle$ in (1) and (2) is an entangled state of the atom-photon system, so it is clear from the start that none of the four (pure) $\psi$-states can be ascribed to the atom alone, as long as the system is in state $|\Psi\rangle$. It is true that this commits the realist to Mohrhoff's as-if-statements (compare my remarks on the matter-density version of GRW in section 2, but to say that reality differs from what measurements seem to reveal is very different from saying (as the operationalist would) that there is no reality beyond measurements. The second thing a reality-of-states view should take seriously is state reduction. After a measurement on one of the two particles, the formalism of standard quantum mechanic: ${ }^{6}$ no longer describes the atom-photon system by $|\Psi\rangle$,

5 I should mention that Mohrhoff has earlier defended a "reality-of-states point of view" himself [18. Therefore, I cannot claim originality for much of what I will have to say in the rest of this section. In response to criticism by Englert et al. [5], Mohrhoff later abandoned this view in favor of a "reality-of-phenomena point of view", which may be seen as a kind of compromise between the minimalistic interpretation advocated by Englert et al. and realism about the quantum state. The details of this debate do not matter here, because I am not attempting to evaluate the final positions of either of the two parties, but am only concerned with the claim that quantum state realism is not viable, a claim which seems to be common ground between Englert et al. and Mohrhoff's more recent paper.

6 As is well known, there is no satisfying characterization of "measurement" within standard quantum mechanics [2]. The reality-of-states view presented here is compatible with different solutions to this problem, e.g., the GRW theory or an 
but by a separable state. A realistic view of the quantum state suggests that this change of description corresponds to a real physical change. This implies that the metaphysical significance of the sorting of atoms into subensembles depends on the temporal ordering of the measurements.7 If the photon is measured prior to the atom's arrival at the screen, the subensembles correspond to real properties, because the photon measurement brings about the state reduction

$$
|\Psi\rangle(x) \rightarrow|i\rangle \psi_{i}(x), \quad i \in\{1,2,+,-\},
$$

so that each atom actually is in one of the $\psi_{i}$ states prior to its hitting the screen. But if the time order of the two measurements is reversed, the atom never is in any one of these states, because (3) does not occur. Instead, the atom's arrival at the screen (at a location $x_{0}$ ) results in a state reduction of the form

$$
|\Psi\rangle(x) \rightarrow[\alpha|1\rangle+\beta|2\rangle] \phi_{x_{0}}(x),
$$

where $\phi_{x_{0}}(x)$ is a spatial wave function well localized at $x_{0} 8$ In this case, assigning the atom to a subensemble depending on the result of the photon measurement implies nothing about the physical state of the atom, whether past or present ${ }^{9}$

Everett-type approach. In the latter case, state reduction is to be understood as a branching of worlds due to environment-induced decoherence. Some additional remarks on how this plays out in the experiments discussed here will be given in footnotes 9 and 13

7 This becomes problematic in a relativistic context, where the temporal ordering of spacelike separated events depends on the reference frame. The question of how to reconcile quantum non-locality with relativity is beyond the scope of this paper (see [16 for an insightful discussion). But since an anonymous referee has pressed me on this point, I offer the following justification for assuming a well-defined temporal ordering between any two events: Some of the most widely discussed realistic versions of quantum theory (e.g., Bohmian mechanics and the matterdensity version of GRW) involve a commitment to a preferred foliation of spacetime. If these proposals are reasonable, then so is the assumption that there is a definite (although undetectable) temporal ordering between any two events.

8 For details about the coefficients $\alpha$ and $\beta$, see [5, eq. 8]. Of course, we could equally well express the photon state in the $|+\rangle,|-\rangle$ basis.

9 Although I have derived this result within a view that takes state reductions as real events, it is interesting to note that the result is not peculiar to such a view. Hiley and Callaghan [1] analyze the situation from a Bohmian perspective, which does not regard state reductions as real, but instead assigns a definite trajectory to each atom. Their conclusion is analogous to mine, namely that the retrospective sorting into subensembles does not correspond to differences in the trajectories of the atoms.

From an Everettian perspective, there is a sense in which the subensembles never have any metaphysical significance: Strictly speaking, according to Everettian quantum mechanics, superpositions never disappear, hence if the atom started out as part of an entangled system, it never enters into one of the $\psi_{i}$ states. But in that sense, no experience ever has metaphysical significance, because our experiences keep telling us that the world around us is in some unique, non-superposed state, while it really is not. Everettians usually prefer a less drastic formulation, according to which our experience is not so much illusory as merely incomplete: The macroscopic world we experience then counts as perfectly real, but it is only one branch among infinitely many others which we do not experience. With such a branch-relative notion of reality in place, the metaphysical significance of the measurement subensembles can be assessed in exactly the same way as in a theory with 
But then, isn't the appearance of definite (WW or DS) patterns within the subensembles somewhat miraculous? I do not think so. Given that the atom and the photon formed an entangled system up to the moment of the atom's detection, it is not so surprising that we can obtain interesting patterns by correlating the location of the atom's detection with the result of a posterior photon measurement. But this correlating is something the experimenter needs to $d o$; the correlation is no longer "there", once the transition (4) has occurred.

\section{Delayed-Choice Entanglement Swapping}

We can now apply the foregoing considerations to the process of entanglement swapping, first proposed by Yurke and Stoler [29] (see also [30]). In the simplest case, this involves entangled pairs of two-state systems, which can be conveniently described by introducing the four so-called Bell states:

$$
\left|\psi^{ \pm}\right\rangle=\frac{1}{\sqrt{2}}[|0\rangle|1\rangle \pm|1\rangle|0\rangle], \quad\left|\phi^{ \pm}\right\rangle=\frac{1}{\sqrt{2}}[|0\rangle|0\rangle \pm|1\rangle|1\rangle]
$$

Now consider two independent sources, each one emitting a particle pair in the state $\left|\psi^{-}\right\rangle$. Denoting the two pairs by $(A, B)$ and $(C, D)$ respectively, the state of the complete system is given by

$$
|\Psi\rangle=\left|\psi^{-}\right\rangle_{A B}\left|\psi^{-}\right\rangle_{C D}
$$

This is obviously a separable state, reflecting the fact that the two pairs are mutually independent. But now suppose that particles $B$ and $C$ are sent to the same location, where a Bell measurement is performed on them, such that their joint state is projected onto one of the four Bell states $\left|\psi^{ \pm}\right\rangle_{B C}$, $\left|\phi^{ \pm}\right\rangle_{B C}{ }^{10}$ To see how this affects particles $A$ and $D$, we rewrite equation (5) by expressing $|\Psi\rangle$ in the basis given by the Bell states of the pairs $(A, D)$ and $(B, C)$ :

$|\Psi\rangle=\frac{1}{2}\left[\left|\psi^{+}\right\rangle_{A D}\left|\psi^{+}\right\rangle_{B C}-\left|\psi^{-}\right\rangle_{A D}\left|\psi^{-}\right\rangle_{B C}-\left|\phi^{+}\right\rangle_{A D}\left|\phi^{+}\right\rangle_{B C}+\left|\phi^{-}\right\rangle_{A D}\left|\phi^{-}\right\rangle_{B C}\right]$.

Since the Bell states are orthogonal to each other, the Bell measurement on the $(B, C)$ pair projects the state $|\Psi\rangle$ onto an entangled state of the $(A, D)$ pair, for example:

$$
\left\langle\left.\psi^{+}\right|_{B C} \mid \Psi\right\rangle=\frac{1}{2}\left|\psi^{+}\right\rangle_{A D}
$$

state reduction. If the photon is measured first, branching occurs (due to decoherence), and within each branch, the atom really (in the branch-relative sense) enters into one of the $\psi_{i}$ states. By contrast, if the measurement of the photon is delayed, the atom never is in such a state (not even in the branch-relative sense), because no branching occurs until the atom hits the screen.

10 This is an idealized description. In practice, a Bell measurement is unable to identify all of the four Bell states. For technical reasons, experiments usually focus on the singlet state $\left|\psi^{-}\right\rangle$[20, 13]. 
and analogously for the other Bell states. Thus particles $A$ and $D$ emerge as an entangled pair, although they never interacted with each other ${ }^{11}$

As Asher Peres 21] points out, this procedure can be carried out in a delayed-choice mode, such that the decision to perform a Bell measurement on the $(B, C)$ pair may take place after any measurements on the $(A, D)$ pair ${ }^{12}$ But since particles $A$ and $D$ only become entangled with each other if the $(B, C)$ measurement is actually performed, it seems that "entanglement is produced a posteriori, after the entangled particles have been measured and may no longer exist" [21, p. 139]. We have seen in the introduction that Healey takes this to undermine the idea that entanglement is a physical relation. To support his view, he offers the following reductio ad absurdum:

To hold onto that idea in the context of this experiment would require one to maintain not only that which entanglement relation obtains between a pair of photons at some time, but also whether any such relation then obtains between them, depends on what happens to other independent systems later, after the pair has been absorbed into the environment. [9, p. 759]

There is a clear parallel between this argument and the discussion in section 3, and this might seem to force me into accepting Healey's conclusion. In section 3, my unwillingness to accept retrocausation led me to reject the claim that the atom really went through either one of the slits (even if a WW measurement seems to tell us so). Should it then not also lead me to reject the claim that the $(A, D)$ pair really either was or was not in an entangled state prior to the $(B, C)$ measurement? But such an indefiniteness seems incompatible with the view that entanglement relations are real. This result would be particularly troubling in view of the fact that the notion of an entangled state played a crucial role in the account I defended in section 3 .

Yet, a closer look reveals that the parallels between the two cases do not threaten realism about entanglement. Rather, they can be exploited to refute Healey's argument. In section 3 . I showed that a delayed measurement of the photon results in a sorting of atoms into subensembles which do not correspond to any physical properties of the atoms. Precisely the same thing can happen in entanglement swapping: The Bell measurement on the $(B, C)$ pair allows us to sort the $(A, D)$ pairs into four subensembles corresponding to the four Bell states. Without delayed choice, this has physical significance, because each $(A, D)$ pair really is in such a state after the $(B, C)$ measurement ${ }^{13}$ But if the $(A, D)$ measurements precede the $(B, C)$ measurement, the $(A, D)$ pair never is in any of these states. This is entirely compatible with the fact that evaluating the $(A, D)$ measurements within a certain

\footnotetext{
11 It has been argued 24] that the phenomenon of entanglement swapping by itself (even without adding the delayed-choice condition with which I will be concerned in the following) calls into question the "ontological robustness" of entanglement. See [27] for a response to this kind of argument.

12 The proposal has recently been experimentally realized [14.

13 In an Everettian context, "really" here again has to be understood in a branchrelative sense. Analogously to the situation described in footnote 9 the important contrast is that in the delayed-choice case, the $(A, D)$ pair never (not even in a branch-relative sense) is in a pure state.
} 
subensemble shows Bell-type correlations, just as the subensembles in section 3 showed interference or WW patterns.

Therefore, far from being committed to any indeterminacy about entanglement (or any backward-in-time influences), a realistic view of the quantum state yields a perfectly clear assessment of what happens in entanglement swapping: If the $(B, C)$ measurement occurs at a time the complete system is still in state $|\Psi\rangle$, it confers entanglement on the $(A, D)$ pair, if it occurs at a later time, it does not ${ }^{14}$

\section{Conclusion}

I have argued that delayed-choice experiments do not undermine a realistic view of the quantum state. In the case of the double slit, we saw that they merely undermine a simplistic realism which unreflectively identifies the result of a WW experiment with a statement about which slit the particle went through. The quantum eraser and the case of delayed-choice entanglement swapping required a more careful treatment, because one needs to get clear about the metaphysical significance of the subensembles appearing in these experiments. Once this is achieved, a straightforward reality-of-states story can be told for these seemingly troubling cases.

This does, of course, not exclude non-realism about the quantum state. But it seems to me that the empirical success of quantum mechanics gives us at least some prima facie reason to view quantum states as describing an independent reality. The non-realist then needs an argument for the claim that this is a mistake. Wheeler, Englert et al., Mohrhoff and Healey all think that delayed-choice experiments furnish such an argument. This I have shown not to be the case.

The same dialectic applies, more specifically, to the metaphysics of entanglement. The various things physicists can $d o$ with entanglement support the intuition that there must be some reality to it. Against this, Healey argues that delayed-choice entanglement swapping implies an indeterminacy of entanglement which is incompatible with realism. Having shown that the realist can avoid such an indeterminacy, I conclude that we can, until further notice, consider entanglement as a real relation.

Acknowledgements I am grateful to the audiences at the BSPS annual conference (Stirling, July 2012) and the PSA biennial conference (San Diego, November 2012) for inspiring discussions. I also wish to thank Michael Esfeld, Vincent Lam, Jakob Sprickerhof, Stevie Turkington, Charles Sebens and two anonymous referees for many helpful comments.

14 At the time of revising this article, Megidish et al. 17] reported a rather spectacular variant of a delayed-choice entanglement-swapping experiment, in which the photons $A$ and $D$ not only never interact, but never even coexist. My analysis straightforwardly applies to this case as well: Since the complete system is not in state $|\Psi\rangle$ at the time the $(B, C)$ measurement occurs (in fact, there is no fourparticle system at any time in this experiment), no actual entanglement swapping takes place. 


\section{References}

1. Allori, V., Goldstein, S., Tumulka, R., Zanghì, N.: On the common structure of Bohmian mechanics and the Ghirardi-Rimini-Weber theory. British Journal for the Philosophy of Science 59, 353-389 (2008)

2. Bell, J.S.: Against 'measurement'. In: A.I. Miller (ed.) Sixty-two years of uncertainty: historical, philosophical, and physical inquiries into the foundations of quantum mechanics. Plenum Press, New York (1990). Reprinted in J.S. Bell: Speakable and Unspeakable in Quantum Mechanics, 2nd ed., pp. 213231. Cambridge University Press, Cambridge (2004)

3. Clifton, R.: The subtleties of entanglement and its role in quantum information theory. Philosophy of Science 69, S150-S167 (2002)

4. Dürr, D., Goldstein, S., Zanghì, N.: Bohmian mechanics and the meaning of the wave function. In: R. Cohen, M. Horne, J. Stachel (eds.) Experimental Metaphysics: Quantum Mechanical Studies for Abner Shimony, Boston Studies in the Philosophy of Science, vol. 193, pp. 25-38. Kluwer Academic Publishers (1997)

5. Englert, B.G., Scully, M.O., Walther, H.: Quantum erasure in double-slit interferometers with which-way detectors. American Journal of Physics 67, 325-329 (1999)

6. Esfeld, M.: Quantum entanglement and a metaphysics of relations. Studies in History and Philosophy of Modern Physics 35, 601-617 (2004)

7. Feynman, R.P., Leighton, R.B., Sands, M.: The Feynman lectures on physics, vol. 3. Addison-Wesley, Reading MA (1965)

8. French, S.: Individuality, supervenience and Bell's theorem. Philosophical Studies 55, 1-22 (1989)

9. Healey, R.: Quantum theory: A pragmatist approach. British Journal for the Philosophy of Science 63, 729-771 (2012)

10. Hiley, B.J., Callaghan, R.E.: Delayed-choice experiments and the Bohm approach. Physica Scripta 74, 336-348 (2006)

11. Hiley, B.J., Callaghan, R.E.: What is erased in the quantum erasure? Foundations of Physics 36, 1869-1883 (2006)

12. Horodecki, R., Horodecki, P., Horodecki, M., Horodecki, K.: Quantum entanglement. Reviews of Modern Physics 81, 865-942 (2009)

13. Jennewein, T., Weihs, G., Pan, J.W., Zeilinger, A.: Experimental nonlocality proof of quantum teleportation and entanglement swapping. Physical Review Letters 88, 017,903 (2002)

14. Ma, X.s., Zotter, S., Kofler, J., Ursin, R., Jennewein, T., Brukner, Č., Zeilinger, A.: Experimental delayed-choice entanglement swapping. Nature Physics 8, 480-485 (2012)

15. Maudlin, T.: Part and whole in quantum mechanics. In: E. Castellani (ed.) Interpreting Bodies: Classical and Quantum Objects in Modern Physics, pp. 46-60. Princeton University Press, Princeton (1998)

16. Maudlin, T.: Quantum Non-Locality and Relativity, 3rd edn. Wiley-Blackwell, Chichester (2011)

17. Megidish, E., Halevy, A., Shacham, T., Dvir, T., Dovrat, L., Eisenberg, H.S.: Entanglement swapping between photons that have never coexisted. Physical Review Letters 110, 210,403 (2013)

18. Mohrhoff, U.: Restoration of interference and the fallacy of delayed choice: Concerning an experiment proposed by Englert, Scully, and Walter. American Journal of Physics 64, 1468-1475 (1996)

19. Mohrhoff, U.: Objectivity, retrocausation, and the experiment of Englert, Scully, and Walther. American Journal of Physics 67, 330-335 (1999)

20. Pan, J.W., Bouwmeester, D., Weinfurter, H., Zeilinger, A.: Experimental entanglement swapping: Entangling photons that never interacted. Physical Review Letters 80, 3891-3894 (1998)

21. Peres, A.: Delayed choice for entanglement swapping. Journal of Modern Optics 47, 139-143 (2000) 
22. Scully, M.O., Drühl, K.: Quantum eraser: A proposed photon correlation experiment concerning observation and "delayed choice" in quantum mechanics. Physical Review A 25, 2208-2213 (1982)

23. Scully, M.O., Englert, B.G., Walther, H.: Quantum optical tests of complementarity. Nature 351, 111-116 (1991)

24. Seevinck, M.: The quantum world is not built up from correlations. Foundations of Physics 36, 1573-1586 (2006)

25. Teller, P.: Relational holism and quantum mechanics. British Journal for the Philosophy of Science 37, 71-81 (1986)

26. Timpson, C.G.: Information, immaterialism, instrumentalism: Old and new in quantum information. In: A. Bokulich, G. Jaeger (eds.) Philosophy of Quantum Information and Entanglement, pp. 208-227. Cambridge University Press, Cambridge (2010)

27. Timpson, C.G., Brown, H.R.: Building with quantum correlations. Quantum Information Processing 9, 307-320 (2010)

28. Wheeler, J.A.: The 'past' and the 'delayed-choice' double-slit experiment. In: A.R. Marlow (ed.) Mathematical Foundations of Quantum Theory, pp. 9-48. Academic Press, New York (1978)

29. Yurke, B., Stoler, D.: Einstein-Podolsky-Rosen effects from independent particle sources. Physical Review Letters 68, 1251-1254 (1992)

30. Żukowski, M., Zeilinger, A., Horne, M.A., Ekert, A.K.: "Event-ready-detectors" Bell experiment via entanglement swapping. Physical Review Letters 71, 4287$4290(1993)$ 